\title{
Stability Analysis of Predator-Prey Model Using Holling Type II with Time Delay in Stabilization Pond
}

\author{
Gesti Essa Waldhani ${ }^{1}$, Sunarsih ${ }^{2}$, Titi Udjani ${ }^{3}$ \\ ${ }^{1,2,3}$ Department of Mathematics, Faculty of Science and Mathematics, Diponegoro University, Indonesia \\ Corresponding email: gestiwaldhani@rocketmail.com
}

\begin{abstract}
In this paper, we discuss the predator-prey model using Holling type II functional response with the time delay in facultative stabilization pond. In this research, we discuss the predator-prey model using Holling type II functional response with the time delay, determining the equilibrium point, the stability analysis of predator-prey model using Holling type II functional response with the time delay and numerical simulation of the predatorprey model using Holling type II functional response with the time delay. The method used to analyse the problem is by literature study. The steps used are the development of a mathematical model of change of dissolved oxygen concentration, phytoplankton and zooplankton, mathematical equation solving algorithm, field data, and simulation using Maple and Mathematica 9 software and validation with research.
\end{abstract}

Key words: Stability; Predator-prey; Holling II; Time delay; Hopf bifurcation.

\section{Introduction}

The research of the interaction of preypredator will be done analysing the mathematical model. Our model based on the basic of Petrovskii (2015) research which we expanded taking into account zooplankton and considering the influence of predatphytoplankton-oxygen model of Sekerci anion on oxygen dynamics and adding the time delay. This model will be applied facultative stabilization pond. Hull et al. (2008) investigated seasonal and daily dynamics of dissolved oxygen measurements in Mediterranean coastal lagoons. Another plankton-oxygen model has been proposed and analysed by Misra (2010) including the effect of some 'exogenous' factors (such as light, wind intensity, temperature, phosphorus, eutrophication, etc.), hence leaving the internal plankton-oxygen dynamics out of the focus. Misra (2011) proposes and analyses a non-linear mathematical model for algal bloom in a lake to account for the delay in conversion of detritus into nutrients. It is assumed that there is a continuous inflow of nutrients in the lake due to agricultural run-off.

\section{Model Formulation}

\subsection{The baseline model}

We begin with a simple conceptual model that only takes into account the temporal dynamics of the oxygen itself and the phytoplankton as its main producer:

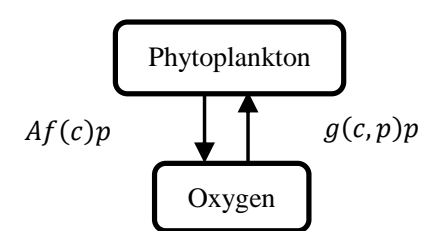

Figure 1. Interactions between oxygen \& phytoplankton

Phytoplankton produces oxygen through photosynthesis during the day-time depending on existence of sunlight and consumes it during the night

$$
\begin{aligned}
& \frac{d c}{d t}=A f(c) p-m c \\
& \frac{d p}{d t}=g(c, p) p
\end{aligned}
$$

here $c$ and $p$ the concentration of the dissolved oxygen and the phytoplankton density $f(c)$ : the amount of oxygen produced per unit time and per unit phytoplankton mass, $g(c, p)$ : the per capita phytoplankton growth rate, $A$ : a coefficient that can take into account the effect of relevant environmental factors and $m c$ : oxygen losses, e.g. due to its diffusion to the atmosphere, plankton breathing, etc. Note that Eq. (1) is linear with respect to $p$ and indeed we are not aware about any evidence that the photosynthesis rate can depend on phytoplankton density. On the contrary, Eq. (2) should normally be nonlinear with respect to $p$ (hence the dependence of $g$ on $p$ ) as the high phytoplankton density is known to damp its growth, e.g. due to self shading and or nutrient depletion. In order to understand what can be the 
properties of functions $f$ and $g$, we have to look more closely at the oxygen production and consumption. Consider $f(c)$ first. Oxygen is produced inside phytoplankton cells in photosynthesis and then diffuse through the cell membrane into the surrounding water. Diffusion flux always directed from areas with higher concentration of the diffusing substance to the areas with lower ones; the larger is the difference between the concentrations, the larger is the flux (cf. the Fick law). Therefore, for the same rate of photosynthesis, the amount of oxygen that gets through the cell membrane will be the larger the lower is the oxygen concentration in the surrounding water. Therefore, $f$ should be a monotonously decreasing function of $c$. We further assume that the oxygen flux through the cell membrane tends to zero when the oxygen concentration in the water is very large, i.e., in physical terms, is close to its saturating value $c \rightarrow \infty$. The above features are qualitatively taken into account by the following parameterization:

$$
f(c)=1-\frac{c}{c+c_{0}}
$$

where $c_{0}$ : the half-saturation constant.

Considering phytoplankton multiplication, we assume that $g(c, p)=\alpha(c)-\gamma p$ where $\alpha(c)$ : the phytoplankton linear growth and $\gamma p$ : intraspecific competition for resources. Eq. (2) for the phytoplankton growth is therefore essentially the logistic growth equation where $\frac{1}{\gamma}$ : plays the role of the carrying capacity, which we assume does not depend on $c$. However, the linear growth rate $\alpha$ should depends on $c$, which can be seen from the following argument. Phytoplankton produce oxygen in photosynthesis during the daytime, but it needs oxygen for breathing during the night; therefore, a low oxygen concentration is unfavorable for phytoplankton and is likely to depress its reproduction. On the other hand, a phytoplankton cell cannot take more oxygen than it needs. Hence $\alpha$ should be monotonously increasing function of $c$ tending to a constant value for $c \rightarrow \infty$. The simplest parameterization for $\alpha$ is then given by the Monod function, so that for $g(c, p)$ we obtain:

$$
g(c, p)=\frac{B c}{c+c_{1}}-\gamma p
$$

where $c_{1}$ : the half-saturation constant and $B$ : the phytoplankton maximum per capita growth rate. With Eqs. (3-4), Eqs. (1-2) take the following form.

$$
\begin{gathered}
\frac{d c}{d t}=A\left(1-\frac{c}{c+c_{0}}\right) p-m c, \\
\frac{d p}{d t}=\left(\frac{B c}{c+c_{1}}-\gamma p\right) p . \\
t^{\prime}=t m, c^{\prime}=\frac{c}{c_{0}}, p^{\prime}=\frac{\gamma p}{m}, \hat{A}=\frac{A}{c_{0} \gamma^{\prime}} \\
\hat{B}=\frac{B}{m}, \widehat{c_{1}}=\frac{c_{1}}{c_{0}} .
\end{gathered}
$$

Thus, Eqs. (5-6) is equivalent to the following equation.

$$
\begin{aligned}
& \frac{d c}{d t}=A\left(1-\frac{c}{c+c_{0}}\right) p-m c, \\
& \frac{d p}{d t}=\left(\frac{B c}{c+c_{1}}-p\right) p .
\end{aligned}
$$

Eqs. (5-6) have two equilibrium points, i.e. $T_{0}(0,0)$ and $T_{1}\left(\frac{-c_{1}-1+\sqrt{\Omega}}{2}, \frac{2 A B+c_{1}\left(c_{1}-1\right)-c_{1} \sqrt{\Omega}}{2 A}\right)$ where $\Omega=4 \mathrm{AB}+\left(c_{1}-1\right)^{2}$ with the conditions $2 A B+c_{1}\left(c_{1}-1\right)>0$ and $A B>c_{1}$ with Jacobian matrix $J=$ $\left(\begin{array}{cc}-\frac{A p}{(c+1)^{2}}-1 & \frac{A}{c+1} \\ \frac{B c_{1} p}{\left(c+c_{1}\right)^{2}} & \frac{B c}{c+c_{1}}-2 p\end{array}\right)$. From the Jacobian matrix, Eqs. (5-6) calculated at each equilibrium point obtaining the eigenvalues $\lambda_{1}=0$ and $\lambda_{2}=$ 1 for $T_{0}, \lambda_{1,2}=\frac{-\rho \pm \sqrt{\rho^{2}-4 \Omega}}{2}$ where $\rho=\frac{A \tilde{p}}{(\tilde{c}+1)^{2}}-$ $\frac{B \tilde{c}}{\tilde{c}+c_{1}}+2 \tilde{p}+1 \quad$ and $\quad \Omega=$ $\frac{\tilde{p} \tilde{c}\left(2 \tilde{p} \tilde{c} A+4 \tilde{p} A c_{1}+2 \tilde{c}^{3}+4 \tilde{c}^{2}+2 \tilde{c}+4 \tilde{c}^{2} c_{1}+8 \tilde{c} c_{1}+4 c_{1}+2 \tilde{c} c_{1}{ }^{2}+4 c_{1}{ }^{2}\right)}{(\tilde{c}+1)^{2}\left(\tilde{c}+c_{1}\right)^{2}}$

$$
\begin{aligned}
& +\frac{B \tilde{c}\left(-\tilde{c}^{3}-2 \tilde{c}^{2}-\tilde{c}-\tilde{c}^{2} c_{1}-2 \tilde{c} c_{1}-c_{1}+\tilde{p} \tilde{c} A\right)+\tilde{p} c_{1}\left(2 \tilde{p} c_{1} A-A B+2 c_{1}\right)}{(\tilde{c}+1)^{2}\left(\tilde{c}+c_{1}\right)^{2}} \text { for } \\
& T_{1}\left(\frac{-c_{1}-1+\sqrt{\Omega}}{2}, \frac{2 A B+c_{1}\left(c_{1}-1\right)-c_{1} \sqrt{\Omega}}{2 A}\right) \text { where } \\
& \Omega=4 A B+\left(c_{1}-1\right)^{2} \text {. }
\end{aligned}
$$

The simulation results can be shown in Figure 2.
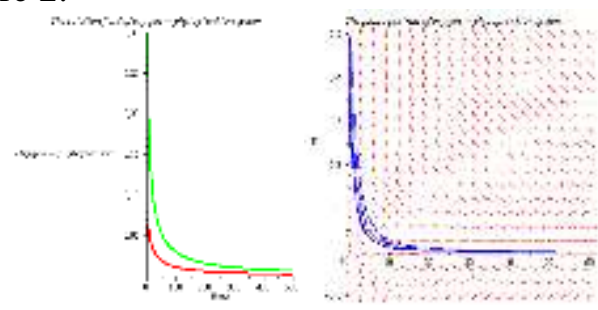

Figure 2. The Simulation Results

The solution field and phase portrait of the oxygen-phytoplankton system at the equilibrium point

$T_{1}\left(\frac{-c_{1}-1+\sqrt{\Omega}}{2}, \frac{2 A B+c_{1}\left(c_{1}-1\right)-c_{1} \sqrt{\Omega}}{2 A}\right)$ where $\Omega=$ $4 A B+\left(c_{1}-1\right)^{2}$ with $2 A B+c_{1}\left(c_{1}-1\right)>0$ and $A B>c_{1}$

\subsection{The 'advanced' three-component model}

The corresponding model is described by the following differential equations: 


$$
\begin{aligned}
& \frac{d c}{d t}=A f(c) p-c, \\
& \frac{d p}{d t}=g(c, p) p-e(p, z), \\
& \frac{d z}{d t}=e(p, z)-\mu z,
\end{aligned}
$$

where all notations are the same as in section 2.1. Additionally, here $z$ : the zooplankton density at time $t$, and the function of $e(p, z)$ : the per capita zooplankton growth rate due to predation where $\mu$ : the zooplankton mortality rate. In the model above, we assume that the phytoplankton-zooplankton interaction is described by the standard prey-predator model with functional response of Holling type II. The second negative term of Eq. (10) corresponds to the grazing of zooplankton on phytoplankton, hence this predation contributes to predator (zooplankton) growth term $\beta e(p, z)$. We consider a Holling type II predator response and use the following parametrization for predation:

$$
e(p, z)=\frac{\beta p z}{p+h}
$$

where $h$ : the half-saturation constant and $\beta$ (dimensionless): maximum per capita growth rate of zooplankton. With Eq. (1) and Eqs. (7-8), then Eqs. (9-11) take the following form:

$$
\begin{aligned}
& \frac{d c}{d t}=A\left(1-\frac{c}{c+1}\right) p-c, \\
& \frac{d p}{d t}=\left(\frac{B c}{c+c_{1}}-p\right) \gamma p-\frac{\beta p z}{p+h^{\prime}} \\
& \frac{d z}{d t}=\frac{\beta p z}{p+h}-\mu z . \\
& t^{\prime}=t m, c^{\prime}=\frac{c}{c_{0}}, p^{\prime}=\frac{\gamma p}{m}, z^{\prime}=\frac{\beta z}{m} \\
& \hat{A}=\frac{A}{c_{0} \gamma}, \hat{B}=\frac{B}{m}, \widehat{c_{1}}=\frac{c_{1}}{c_{0}}, h^{\prime}=\frac{\gamma h}{m}, \hat{\mu}=\frac{\mu}{m} .
\end{aligned}
$$

Thus, Eqs. (13-15) is equivalent to the following equation.

$$
\begin{aligned}
& \frac{d c}{d t}=A\left(1-\frac{c}{c+1}\right) p-c, \\
& \frac{d p}{d t}=\left(\frac{B c}{c+c_{1}}-p\right) p-\frac{p z}{p+h}, \\
& \frac{d z}{d t}=\frac{\beta p z}{p+h}-\mu z .
\end{aligned}
$$

Then by giving the discrete time-delay in the growth rate of the predator population, the equation models become

$$
\begin{aligned}
& \frac{d c(t)}{d t}=A\left(1-\frac{c(t)}{c(t)+1}\right) p(t)-c(t), \\
& \frac{d p(t)}{d t}=\left(\frac{B c(t)}{c(t)+c_{1}}-p(t)\right) p(t)-\frac{p(t) z(t)}{p(t)+h}, \\
& \frac{d z(t)}{d t}=\frac{\beta p(t-\tau) z(t)}{p(t-\tau)+h}-\mu z(t) .
\end{aligned}
$$

\section{Equilibria}

\section{Theorem 1.}

From the above Eqs. (16-18), we obtain:

1. Without the condition, Eqs. (16-18) have one equilibrium points i.e. the equilibrium point $T_{0}(0,0,0)$.
2. If $2 A B+c_{1}\left(c_{1}-1\right)>0$ and $A B>c_{1}$ then Eqs. (16-18) have two the equilibrium points i.e. the equilibrium point $T_{0}(0,0,0) \quad$ and $T_{1}\left(\frac{-c_{1}-1+\sqrt{\Omega}}{2}, \frac{2 A B+c_{1}\left(c_{1}-1\right)-c_{1} \sqrt{\Omega}}{2 A}, 0\right)$ where $\Omega=4 A B+\left(c_{1}-1\right)^{2}$.

3. If $\beta>\mu$ and $\frac{B\left(-1+\sqrt{1+\frac{4 A \mu h}{\beta-\mu}}\right)}{-1+\sqrt{1+\frac{4 A \mu h}{\beta-\mu}+2 c_{1}}}>\frac{\mu h}{\beta-\mu}$ then Eqs. (16-18) have two equilibrium points i.e. the equilibrium point $T_{0}(0,0,0)$ and

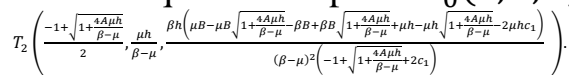

4. If $2 A B+c_{1}\left(c_{1}-1\right)>0, A B>c_{1}, \beta>$ $\mu$ and $\frac{B\left(-1+\sqrt{1+\frac{4 A \mu h}{\beta-\mu}}\right)}{-1+\sqrt{1+\frac{4 A \mu h}{\beta-\mu}+2 c_{1}}}>\frac{\mu h}{\beta-\mu}$ then Eqs. (16-18) have three equilibrium points i.e. the equilibrium point $T_{0}(0,0,0), T_{1}\left(\frac{-c_{1}-1+\sqrt{\Omega}}{2}, \frac{2 A B+c_{1}\left(c_{1}-1\right)-c_{1} \sqrt{\Omega}}{2 A}, 0\right)$

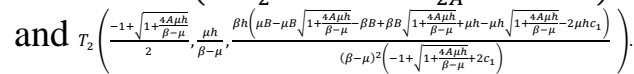

Proof.

Eqs. (16-18) realizes the equilibrium point when

$$
\begin{aligned}
& A\left(1-\frac{c}{c+1}\right) p-c=0, \\
& \left(\frac{B c}{c+c_{1}}-p\right) p-\frac{p z}{p+h}=0, \\
& \frac{\beta p z}{p+h}-\mu z=0 .
\end{aligned}
$$

From Eq. (24) $\frac{\beta \tilde{p} \tilde{z}}{\tilde{p}+h}-\mu \tilde{z}=0 \Rightarrow \tilde{z}=0 \mathrm{~V}$ $\tilde{p}=\frac{\mu h}{\beta-\mu}$.

(1) Case $\tilde{z}=0 .\left(\frac{B \tilde{c}}{\tilde{c}+c_{1}}-\tilde{p}\right) \tilde{p}-\frac{\tilde{p} \tilde{z}}{\tilde{p}+h}=0 \Rightarrow$ $\tilde{p}=0 \vee \tilde{c}=\frac{\tilde{p} c_{1}}{B-\tilde{p}}$.

(a) Case $\tilde{z}=0$ and $\tilde{p}=0 . A\left(1-\frac{\tilde{c}}{\tilde{c}+1}\right) \tilde{p}-$ $\tilde{c}=0 \Leftrightarrow \tilde{c}=0$. So we obtain $T_{0}(0,0,0)$.

(b) Case $\quad \tilde{z}=0 \quad$ and $\quad \tilde{c}=\frac{\tilde{p} c_{1}}{B-\tilde{p}} \cdot A(1-$ $\left.\frac{\tilde{c}}{\tilde{c}+1}\right) \tilde{p}-\tilde{c}=0 \Leftrightarrow \tilde{p}=\frac{\tilde{c}}{A}(\tilde{c}+1)$.

Substituting $\tilde{c}=\frac{\tilde{p} c_{1}}{B-\tilde{p}}$ into the equation $\tilde{p}=\frac{\tilde{c}}{A}(\tilde{c}+1)$ we get $\tilde{p}_{1,2}=\frac{-\sigma \pm \sqrt{\sigma^{2}-4 A \kappa}}{2 A}$, where $\sigma=-2 A B-c_{1}{ }^{2}+c_{1}$ and $\kappa=$ $A B^{2}$

$-B c_{1}$. Considering $\tilde{p}=\frac{-\sigma-\sqrt{\sigma^{2}-4 A \kappa}}{2 A}>0$, then $\sigma^{2}-4 A \kappa \geq 0, \sigma<0$ and $-\sigma-$ $\sqrt{\sigma^{2}-4 A \kappa}>0 \Leftrightarrow-4 A \kappa<0$, such that $\kappa>0 \Leftrightarrow A B>c_{1}$. Considering $\tilde{p}=$ $\frac{-\sigma+\sqrt{\sigma^{2}-4 A \kappa}}{2 A}>0$ then $\sigma^{2}-4 A \kappa \geq$ 
$0, \sigma<0$ and $-\sigma+\sqrt{\sigma^{2}-4 A \kappa}>0 \Leftrightarrow$ $-4 A \kappa>0$, so that $\kappa<0 \Leftrightarrow A B<c_{1}$. Therefore, the unique positive root exists for $\tilde{p}=\frac{-\sigma-\sqrt{\sigma^{2}-4 A \kappa}}{2 A}>0$ with the conditions $\sigma^{2}-4 A \kappa \geq 0, \sigma<0$ and $A B>c_{1}$. So we obtain $T_{1}\left(\frac{\left(\frac{-\sigma-\sqrt{\sigma^{2}-4 A \kappa}}{2 A}\right) c_{1}}{B+\frac{\sigma+\sqrt{\sigma^{2}-4 A \kappa}}{2 A}}, \frac{-\sigma-\sqrt{\sigma^{2}-4 A \kappa}}{2 A}, 0\right)$ with the conditions $\sigma^{2}-4 A \kappa \geq 0, \sigma<0$ and $A B>c_{1}$.

Substituting $\sigma=-2 A B-c_{1}{ }^{2}+c_{1}$ and $\kappa=A B^{2}-B c_{1}$ into the equation $\underline{\left(\frac{-\sigma-\sqrt{\sigma^{2}-4 A \kappa}}{2 A}\right) c_{1}}$

$B+\frac{\sigma+\sqrt{\sigma^{2}-4 A \kappa}}{2 A}$

we

get

$\frac{\left(-\left(-2 A B-c_{1}^{2}+c_{1}\right)-\sqrt{\left(-2 A B-c_{1}{ }^{2}+c_{1}\right)^{2}-4 A\left(A B^{2}-B c_{1}\right)}\right) c_{1}}{2 A B+\left(-2 A B-c_{1}^{2}+c_{1}\right)+\sqrt{\left(-2 A B-c_{1}{ }^{2}+c_{1}\right)^{2}-4 A\left(A B^{2}-B c_{1}\right)}}=$

$\frac{-c_{1}-1+\sqrt{\Omega}}{2}, \quad$ with $\quad \Omega=4 A B+\left(c_{1}-\right.$ $1)^{2}$. Substituting $\sigma=-2 A B-c_{1}{ }^{2}+c_{1}$ and $\kappa=A B^{2}-B c_{1}$ into the equation $\frac{-\sigma-\sqrt{\sigma^{2}-4 A \kappa}}{2 A}$

we

obtain

$\frac{-\left(-2 A B-c_{1}{ }^{2}+c_{1}\right)-\sqrt{\left(-2 A B-C_{1}{ }^{2}+c_{1}\right)^{2}-4 A\left(A B^{2}-B c_{1}\right)}}{2 A}=$

$\frac{2 A B+c_{1}\left(c_{1}-1\right)-c_{1} \sqrt{\Omega}}{2 A}$. So we get $T_{1}\left(\frac{-c_{1}-1+\sqrt{\Omega}}{2}, \frac{2 A B+c_{1}\left(c_{1}-1\right)-c_{1} \sqrt{\Omega}}{2 A}, 0\right)$ wher e $\Omega=4 A B+\left(c_{1}-1\right)^{2}$ with the conditions $2 A B+c_{1}\left(c_{1}-1\right)>0$ and $A B>c_{1}$.

(2) Case $\tilde{p}=\frac{\mu h}{\beta-\mu} . \quad\left(\frac{B \tilde{c}}{\tilde{c}+c_{1}}-\tilde{p}\right) \tilde{p}-\frac{\tilde{p} \tilde{z}}{\tilde{p}+h}=$ $0 \Leftrightarrow \tilde{z}=\left(\frac{\mu h}{\beta-\mu}+h\right)\left(\frac{B \tilde{c}}{\tilde{c}+c_{1}}-\frac{\mu h}{\beta-\mu}\right)$.

Substituting $\tilde{p}=\frac{\mu h}{\beta-\mu}$ into the Eq. (22) we obtain $A\left(1-\frac{\tilde{c}}{\tilde{c}+1}\right) \tilde{p}-\tilde{c}=0 \Rightarrow \tilde{c}_{1,2}=$ $\frac{-1 \pm \sqrt{1+\frac{4 A \mu h}{\beta-\mu}}}{2}$. Then there is $T_{2}\left(\frac{-1+\sqrt{1+\frac{4 A \mu h}{\beta-\mu}}}{2}, \frac{\mu h}{\beta-\mu}, \frac{\beta h\left(\mu B-\mu B \sqrt{1+\frac{4 A \mu h}{\beta-\mu}-\beta B+\beta B} \sqrt{\left.1+\frac{4 A \mu h}{\beta-\mu}+\mu h-\mu h \sqrt{1+\frac{4 A \mu h}{\beta-\mu}-2 \mu h c_{1}}\right)}\right.}{(\beta-\mu)^{2}\left(-1+\sqrt{1+\frac{4 A \mu h}{\beta-\mu}+c_{1}}\right)}\right)$ with the conditions $\quad \beta>\mu \quad$ and $\frac{B\left(-1+\sqrt{1+\frac{4 A \mu h}{\beta-\mu}}\right)}{-1+\sqrt{1+\frac{4 A \mu h}{\beta-\mu}}+2 c_{1}}>\frac{\mu h}{\beta-\mu}$.

\section{Stability Analysis}

Theorem 2.

We have $T_{0}, T_{1}$ and $T_{2}$ which three equilibrium points of the Eqs. (16-18) as in theorem 1 .

1. The equilibrium point $T_{0}$ is not informative.
2. The equilibrium point $T_{1}$ is node stable when $c_{1}=0,4 ; A=0,42$ and node unstable when $c_{1}=0,4 ; A=0,5$ with $B=1 ; \gamma=1 ; \beta=1 ; \mu=0,5 ; h=0,1$.

3. The equilibrium point $T_{2}$ is node stable if only if $-b_{1}-b_{4}-b_{7}>0, b_{1} b_{4}+$ $b_{1} b_{7}+b_{4} b_{7}-b_{2} b_{3}-b_{5} b_{6}>0$ and $b_{2} b_{3} b_{7}-b_{1} b_{4} b_{7}+b_{5} b_{6} b_{1}>0 \quad$ where $b_{1}=\frac{-A \tilde{p}}{(\tilde{c}+1)^{2}}-1, b_{2}=\frac{A}{\tilde{c}+1}, b_{3}=$ $\frac{B c_{1} \tilde{p}}{\left(\tilde{c}+c_{1}\right)^{2}}, b_{4}=\frac{B \tilde{c}}{\tilde{c}+c_{1}}-2 \tilde{p}-\frac{\tilde{z} h}{(\tilde{p}+h)^{2}}, b_{5}=$ $-\frac{\tilde{p}}{\tilde{p}+h}, b_{6}=\frac{\beta h \tilde{z}}{(\tilde{p}+h)^{2}}, b_{7}=\frac{\beta \tilde{p}}{\tilde{p}+h}-\mu$.

Proof.

The general Jacobian matrix of Eqs. (1618) is given by $J=$ $\left(\begin{array}{ccc}\frac{-A p}{(c+1)^{2}}-1 & \frac{A}{c+1} & 0 \\ \frac{B c_{1} p}{\left(c+c_{1}\right)^{2}} & \frac{B c}{c+c_{1}}-2 p-\frac{z h}{(p+h)^{2}} & -\frac{p}{p+h} \\ 0 & \frac{\beta h z}{(p+h)^{2}} & \frac{\beta p}{p+h}-\mu\end{array}\right)$

(1) At $T_{0}(0,0,0)$, the Jacobian matrix is $J(0,0,0)=\left(\begin{array}{ccc}-1 & A & 0 \\ 0 & 0 & 0 \\ 0 & 0 & -\mu\end{array}\right)$. Thus, we get $\lambda_{1}=-1, \lambda_{2}=0$ and $\lambda_{3}=-\mu$.

(2) At $T_{1}(\tilde{c}, \tilde{p}, 0)$, the Jacobian matrix is $J(\tilde{c}, \tilde{p}, 0)=$

$$
\left(\begin{array}{ccc}
-\frac{A \tilde{p}}{(\tilde{c}+1)^{2}}-1-\lambda & \frac{A}{\tilde{c}+1} & 0 \\
\frac{B c_{1} \tilde{p}}{\left(\tilde{c}+c_{1}\right)^{2}} & \frac{B \tilde{c}}{\tilde{c}+c_{1}}-2 \tilde{p}-\lambda & -\frac{\tilde{p}}{\tilde{p}+h} \\
0 & 0 & \frac{\beta \tilde{p}}{\tilde{p}+h}-\mu-\lambda
\end{array}\right) .
$$

Thus, we get $\lambda_{1}=\frac{\beta \tilde{p}}{\tilde{p}+h}-\mu \vee \lambda_{1,2}=$ $\frac{-\kappa \pm \sqrt{\kappa^{2}-4 \Theta}}{2}$ where $\quad \kappa=\frac{A \tilde{p}}{(\tilde{c}+1)^{2}}-\frac{B \tilde{c} \gamma}{\tilde{c}+c_{1}}+$ $2 \tilde{p}+1 \quad$ and $\Theta=$ $\frac{A \tilde{p}\left(-B \tilde{c}^{2}-2 B \tilde{c} c_{1}+2 \tilde{p} \tilde{c}^{2}+4 \tilde{p} \tilde{c} c_{1}+2 \tilde{p} c_{1}{ }^{2}\right)}{(\tilde{c}+1)^{2}\left(\tilde{c}+c_{1}\right)^{2}}+$ $\frac{B\left(\tilde{c}^{4}-2 \tilde{c}^{3}-\tilde{c}^{2}-\tilde{c}^{3} c_{1}-2 \tilde{c}^{2} c_{1}-\tilde{c} c_{1}-A c_{1} \tilde{p}\right)}{(\tilde{c}+1)^{2}\left(\tilde{c}+c_{1}\right)^{2}}+$ $\frac{2 \tilde{p}\left(\tilde{c}^{4}+2 \tilde{c}^{3} c_{1}+\tilde{c}^{2} c_{1}^{2}+2 \tilde{c}^{3}+4 \tilde{c}^{2} c_{1}+2 c_{1}{ }^{2} \tilde{c}+\tilde{c}^{2}+2 c_{1} \tilde{c}\right)}{(\tilde{c}+1)^{2}\left(\tilde{c}+c_{1}\right)^{2}}$.

(3) At $T_{2}(\tilde{c}, \tilde{p}, \tilde{z})$ the Jacobian matrix is $J(\tilde{c}, \tilde{p}, \tilde{z})=$

$$
\begin{aligned}
& \left(\begin{array}{ccc}
\frac{-A \tilde{p}}{(\tilde{c}+1)^{2}}-1 & \frac{A}{\tilde{c}+1} & 0 \\
\frac{B c_{1} \tilde{p}}{\left(\tilde{c}+c_{1}\right)^{2}} & \frac{B \tilde{c}}{\tilde{c}+c_{1}}-2 \tilde{p}-\frac{\tilde{z} h}{(\tilde{p}+h)^{2}} & -\frac{\tilde{p}}{\tilde{p}+h} \\
0 & \frac{\beta h \tilde{z}}{(\tilde{p}+h)^{2}} & \frac{\beta \tilde{p}}{\tilde{p}+h}-\mu
\end{array}\right)= \\
& \frac{A \tilde{p}}{(\tilde{c}+1)^{2}}-1+\frac{B \tilde{c}}{\tilde{c}+c_{1}}-2 \tilde{p}-\frac{\tilde{z} h}{(\tilde{p}+h)^{2}}+ \\
& \frac{\beta \tilde{p}}{\tilde{p}+h}, \varepsilon=-\frac{A \tilde{p} \mu}{(\tilde{c}+1)^{2}}-\mu+\frac{B \tilde{c} \mu}{\tilde{c}+c_{1}}-2 \tilde{p} \mu- \\
& \frac{\tilde{z} h \mu}{(\tilde{p}+h)^{2}}+\frac{A \tilde{p} B \tilde{c}}{(\tilde{c}+1)^{2}\left(\tilde{c}+c_{1}\right)}-\frac{2 \tilde{p}^{2} A}{(\tilde{c}+1)^{2}}- \\
& \frac{A \tilde{p} \tilde{z} h}{(\tilde{c}+1)^{2}(\tilde{p}+h)^{2}}+\frac{B \tilde{c}}{\tilde{c}+c_{1}}-2 \tilde{p}-\frac{\tilde{z} h}{(\tilde{p}+h)^{2}}+
\end{aligned}
$$




$$
\begin{aligned}
& \frac{A \tilde{p}^{2} \beta}{(\tilde{p}+h)(\tilde{c}+1)^{2}}+\frac{\beta \tilde{p}}{\tilde{p}+h}-\frac{\beta \tilde{p} B \tilde{c}}{\left(\tilde{c}+c_{1}\right)(\tilde{p}+h)}+\frac{2 \beta \tilde{p}^{2}}{\tilde{p}+h}+ \\
& \frac{A B c_{1} \tilde{p}}{(\tilde{c}+1)\left(\tilde{c}+c_{1}\right)^{2}}, \rho=-\frac{A \beta \tilde{p}^{2} \tilde{z} h}{(\tilde{c}+1)^{2}(\tilde{p}+h)^{3}}- \\
& \frac{\beta \tilde{p} h \tilde{z}}{(\tilde{p}+h)^{3}}-\frac{A B \beta \tilde{p}^{2} \tilde{c}}{(\tilde{c}+1)^{2}\left(\tilde{c}+c_{1}\right)(\tilde{p}+h)}+ \\
& \frac{2 A \tilde{p}^{3} \beta}{(\tilde{p}+h)(\tilde{c}+1)^{2}}+\frac{A \beta \tilde{p}^{2} \tilde{z} h}{(\tilde{c}+1)^{2}(\tilde{p}+h)^{3}}- \\
& \frac{B \beta \tilde{p} \tilde{c}}{\left(\tilde{c}+c_{1}\right)(\tilde{p}+h)}+\frac{2 \beta \tilde{p}^{2}}{\tilde{p}+h}+\frac{\beta \tilde{p} \tilde{z} h}{(\tilde{p}+h)^{3}}- \\
& \frac{A B \beta \tilde{p}^{2} c_{1}}{(\tilde{c}+1)\left(\tilde{c}+c_{1}\right)^{2}(\tilde{p}+h)}+\frac{A B c_{1} \tilde{p} \mu}{(\tilde{c}+1)\left(\tilde{c}+c_{1}\right)^{2}}- \\
& \frac{2 A \tilde{p}^{2} \mu}{(\tilde{c}+1)^{2}}-\frac{A \tilde{p} \tilde{z} h \mu}{(\tilde{c}+1)^{2}(\tilde{\tilde{c}}+h)^{2}}+\frac{B \tilde{c} \mu}{\tilde{c}+c_{1}}-2 \tilde{p} \mu- \\
& \frac{\tilde{z} h \mu}{(\tilde{p}+h)^{2}}+\frac{A B \tilde{p} \tilde{c} \mu}{(\tilde{c}+1)^{2}\left(\tilde{c}+c_{1}\right)} .
\end{aligned}
$$

So that, we have the eigenvalues $\lambda_{1}=\frac{\phi}{3}+$

$\frac{2^{\frac{1}{3}}\left(-\phi^{2}-3 \varepsilon\right)}{3\left(-27 \rho-2 \phi^{3}-9 \phi \varepsilon+3 \sqrt{3} \sqrt{27 \rho^{2}+4 \rho \phi^{3}+18 \rho \phi \varepsilon-\phi^{2} \varepsilon^{2}-4 \varepsilon^{3}}\right)^{\frac{1}{3}}}$

$-\frac{\left(-27 \rho-2 \phi^{3}-9 \phi \varepsilon+3 \sqrt{3} \sqrt{27 \rho^{2}+4 \rho \phi^{3}+18 \rho \phi \varepsilon-\phi^{2} \varepsilon^{2}-4 \varepsilon^{3}}\right)^{\frac{1}{3}}}{3.2^{\frac{1}{3}}}$,

$\lambda_{2}=\frac{\phi}{3}-$

\begin{tabular}{|c|c|c|c|c|c|c|}
\hline & \multicolumn{5}{|c|}{$A$} \\
\hline & & 0,1 & 0,2 & 0,5 & 0,9 & 1 \\
\hline \multirow{16}{*}{$c_{1}$} & \multirow{3}{*}{1} & $\begin{array}{l}1,66227766 \\
+2566655867\end{array}$ & $\begin{array}{l}0,7360679785 \\
+15855384 i\end{array}$ & $\begin{array}{l}-0,0857864380 \\
+0,579470824\end{array}$ & $-0,00661091$ & 0 \\
\hline & & $\begin{array}{l}1,66227766 \\
-2,566655867\end{array}$ & $\begin{array}{l}0,7360679785 \\
-1,5855384 i\end{array}$ & $\begin{array}{l}-0,0857864380 \\
-0,579470824 i\end{array}$ & $-0,88520397$ & -1 \\
\hline & & 0,548490076 & $\begin{array}{l}1,0303004 i \\
0,588022902\end{array}$ & 0,818254882 & $\frac{-0,08520991}{-1,67829583}$ & $-0,5$ \\
\hline & \multirow{4}{*}{0,9} & $\begin{array}{l}1,516484013 \\
+2665100693\end{array}$ & $\begin{array}{l}0,625 \\
+155623745 i\end{array}$ & $\begin{array}{l}-0,1581425466 \\
+0474563198\end{array}$ & \begin{tabular}{l|l}
6 \\
63
\end{tabular} & $-0,00503747$ \\
\hline & & 1,516484013 & 0,625 & $-0,1581425466$ & & \\
\hline & & $\begin{array}{r}-2,665100693 \\
\end{array}$ & $-1,55623745 i$ & $\begin{array}{r}-0,4745631983 \\
\end{array}$ & -1 & $-1,09758901$ \\
\hline & & 0,544814796 & 0,586956522 & 0,875981944 & $-0,5$ & $-0,14987518$ \\
\hline & \multirow{3}{*}{0,5} & $\begin{array}{l}0,8983466555 \\
+2,351395796 \\
\end{array}$ & $\begin{array}{r}0,1088032685 \\
+1,044583016 \\
\end{array}$ & 0 & $-0,11462255$ & $-0,14519392$ \\
\hline & & $\begin{array}{l}0,8983466555 \\
-2351395796\end{array}$ & $\begin{array}{l}0,1088032685 \\
-104458301\end{array}$ & -1 & -138914825 & -143364146 \\
\hline & & 0,546177338 & 0,624089671 & $-0,5$ & 0,2596568401 & 0,282425079 \\
\hline & \multirow{3}{*}{0,2} & $\begin{array}{l}-0,040588292 \\
+0,866953062\end{array}$ & 0 & $-0,246179149$ & $-0,43257148$ & $-0,46265589$ \\
\hline & & $\begin{array}{l}-0,040588292 \\
-0,866953062\end{array}$ & -1 & $-1,444051634$ & -155023338 & -156490455 \\
\hline & & 0,638926727 & $-0,5$ & 0,337408543 & $\begin{array}{r}1,3721749596 \\
0,372\end{array}$ & 0,375713622 \\
\hline & \multirow{3}{*}{0,1} & 0 & $-0,181015971$ & $-0,4838571701$ & $-0,63456960$ & $-0,65747993$ \\
\hline & & -1 & $-1,354625205$ & $-1,482206823$ & $-1,53209706$ & $-1,54127589$ \\
\hline & & $-0,5$ & 0,3207196333 & 0,3812872434 & 0,3928571429 & 0,394218114 \\
\hline & & & & $A$ & & \\
\hline & & $\begin{array}{l}0,1 \\
\end{array}$ & 0,2 & $\begin{array}{l}0,5 \\
\end{array}$ & $\begin{array}{l}0,9 \\
\end{array}$ & 1 \\
\hline \multirow{17}{*}{$c_{1}$} & \multirow{3}{*}{1} & $-1,02117347$ & $-1,040657271$ & $-1,091237197$ & $-1,145568145$ & $-1,15742328$ \\
\hline & & $-0,2304756352$ & $-0,212166544$ & $-0,1620324663$ & $-0,0983893376$ & $-0,08100892$ \\
\hline & & 0,09674667318 & 0,09320487245 & 0,08049523843 & 0,05559742367 & 0,046472110 \\
\hline & \multirow{3}{*}{0,9} & $-1,02237922$ & $-1,042834909$ & $-1,095399791$ & $-1,151122063$ & $-1,16319123$ \\
\hline & & $-0,228350907$ & $-0,2081066178$ & $-0,152601415$ & $-0,0790918906$ & $-0,05614276$ \\
\hline & & 0,09636648285 & 0,09236855925 & 0,07762984998 & 0,04575586252 & 0,031605415 \\
\hline & \multirow{3}{*}{0,5} & $-1,031646548$ & $-1,059015298$ & $-1,123938785$ & $-1,186178883$ & $-1,19893930$ \\
\hline & & $-0,2116257137$ & $-0,1763038193$ & $-0,0705023818$ & $\begin{array}{l}0,01535615155 \\
+0.0970068827\end{array}$ & $-1,19893930$ \\
\hline & & 0,09317706039 & 0,08495600762 & $\frac{-0,0 / 5023811}{0,04245685205}$ & $\begin{array}{l}+0,09 / 0068882 \\
0,2596568401\end{array}$ & 0,282425079 \\
\hline & \multirow{4}{*}{0,2} & $-1,058673585$ & $-1,101121087$ & $-1,181140174$ & $-1,239343172$ & $-1,24970633$ \\
\hline & & -0157516010 & -00628160897 & 0,04095695807 & 0,07969248982 & 0,086429988 \\
\hline & & $-0,15 / 516010$ & $-0,0628160827$ & $\frac{-0,137204142}{0,04095695807}$ & $\begin{array}{l}+0,1888958923 \\
0,07969248982\end{array}$ & $\begin{array}{c}-0,19739445 \\
0,086429988\end{array}$ \\
\hline & & 0,07997181851 & 0,03935748338 & $\begin{array}{l}-0,137204142 \\
\end{array}$ & $\begin{array}{l}-0,1888958923 \\
\end{array}$ & $-0,19739445$ \\
\hline & \multirow{4}{*}{0,1} & $-1,092970015$ & $-1,144911751$ & $-1,217140385$ & $-1,253629125$ & $-1,25922614$ \\
\hline & & & 0,02883346866 & 0,09155959672 & 0,1269135054 & 0,132178230 \\
\hline & & $-0,0599416719$ & $+0,115798406$ & $\begin{array}{l}+0,198794181 \\
\end{array}$ & $+0,2446811201$ & $+0,25277389$ \\
\hline & & 0,03815584751 & $\begin{array}{r}0,02883346866 \\
-0,115798406\end{array}$ & $\begin{array}{c}0,09155959672 \\
-0,198794181\end{array}$ & $\begin{array}{l}0,1269135054 \\
-0,2446811201\end{array}$ & $\begin{array}{l}0,132178230 \\
-0,25277389\end{array}$ \\
\hline
\end{tabular}

$(1+i \sqrt{3})\left(-\phi^{2}-3 \varepsilon\right)$

$3.2^{\frac{2}{3}}\left(-27 \rho-2 \phi^{3}-9 \phi \varepsilon+3 \sqrt{3} \sqrt{27 \rho^{2}+4 \rho \phi^{3}+18 \rho \phi \varepsilon-\phi^{2} \varepsilon^{2}-4 \varepsilon^{3}}\right)^{\frac{1}{3}}$

$+\frac{(1-i \sqrt{3})\left(-27 \rho-2 \phi^{3}-9 \phi \varepsilon+3 \sqrt{3} \sqrt{27 \rho^{2}+4 \rho \phi^{3}+18 \rho \phi \varepsilon-\phi^{2} \varepsilon^{2}-4 \varepsilon^{3}}\right)^{\frac{1}{3}}}{\operatorname{li}^{\frac{1}{3}}}$

$\lambda_{3}=\frac{\phi}{3}-$

$\frac{(1-i \sqrt{3})\left(-\phi^{2}-3 \varepsilon\right)}{3.2^{\frac{2}{3}}\left(-27 \rho-2 \phi^{3}-9 \phi \varepsilon+3 \sqrt{3} \sqrt{27 \rho^{2}+4 \rho \phi^{3}+18 \rho \phi \varepsilon-\phi^{2} \varepsilon^{2}-4 \varepsilon^{3}}\right)^{\frac{1}{3}}}$
$+\frac{(1+i \sqrt{3})\left(-27 \rho-2 \phi^{3}-9 \phi \varepsilon+3 \sqrt{3} \sqrt{27 \rho^{2}+4 \rho \phi^{3}+18 \rho \phi \varepsilon-\phi^{2} \varepsilon^{2}-4 \varepsilon^{3}}\right)^{\frac{1}{3}}}{6.2^{\frac{1}{3}}}$.

Table 1. Eigenvalues for $T_{1}(\tilde{c}, \tilde{p}, 0)$ and $T_{2}(\tilde{c}, \tilde{p}, \tilde{z})$

\section{Theorem 3}

Suppose that the conditions $\beta>\mu$ and $\frac{B\left(-1+\sqrt{1+\frac{4 A \mu h}{\beta-\mu}}\right)}{-1+\sqrt{1+\frac{4 A \mu h}{\beta-\mu}+2 c_{1}}}>\frac{\mu h}{\beta-\mu}$ are satisfied and given

$\frac{1}{\omega_{k}} \tan ^{-1}\left(\frac{-b_{4} \omega_{k}{ }^{3}-b_{7} \omega_{k}{ }^{3}-b_{2} b_{3} b_{7} \omega_{k}-b_{1}{ }^{2} b_{4} \omega_{k}-b_{1}{ }^{2} b_{7} \omega_{k}+b_{1} b_{2} b_{3} \omega_{k}}{b_{1}{ }^{2} \omega_{k}{ }^{2}+b_{1} b_{2} b_{3} b_{7}-b_{1}{ }^{2} b_{4} b_{7}+\omega^{4}{ }^{2} \omega_{k} b_{4} b_{7}+b_{2} b_{3} \omega_{k}{ }^{2}}\right)+$ $\frac{2 k \pi}{\omega_{k}}, k=0,1,2, \cdots$, where $\omega_{k}>0$ is obtained from the equation $\omega_{k}=\sqrt{z^{*}}$ where $z^{3}+$ $B z^{2}+C z+D=0$ and $\omega^{6}+B \omega^{4}+$ $C \omega^{2}+D=0$, with $B=b_{1}{ }^{2}+b_{4}{ }^{2}+$ $b_{7}{ }^{2}+2 b_{2} b_{3}, C=-b_{5}{ }^{2} b_{6}{ }^{2}-$ $2 b_{1} b_{4} b_{2} b_{3}+b_{1}{ }^{2} b_{4}{ }^{2}+2 b_{7}{ }^{2} b_{2} b_{3}+$ $b_{4}{ }^{2} b_{7}{ }^{2}+b_{2}{ }^{2} b_{3}{ }^{2}+b_{1}{ }^{2} b_{7}{ }^{2}, D=$ $-2 b_{2} b_{3} b_{7}{ }^{2} b_{1} b_{4}+b_{1}{ }^{2} b_{4}{ }^{2} b_{7}{ }^{2}+$ $b_{2}{ }^{2} b_{3}{ }^{2} b_{7}{ }^{2}-b_{5}{ }^{2} b_{6}{ }^{2} b_{1}{ }^{2}-b_{5}{ }^{2} b_{6}{ }^{2} b_{1}{ }^{2}$ with the conditions $-b_{1}-b_{4}-b_{7}>$ $0, b_{1} b_{4}+b_{1} b_{7}+b_{4} b_{7}-b_{2} b_{3}-b_{5} b_{6}>$ $0, b_{2} b_{3} b_{7}-b_{1} b_{4} b_{7}+b_{5} b_{6} b_{1}>0$ where $b_{1}=\frac{-A \tilde{p}}{(\tilde{c}+1)^{2}}-1, b_{2}=\frac{A}{\tilde{c}+1}, b_{3}=$ $\frac{B c_{1} \tilde{p}}{\left(\tilde{c}+c_{1}\right)^{2}}, b_{4}=\frac{B \tilde{c}}{\tilde{c}+c_{1}}-2 \tilde{p}-\frac{\tilde{z} h}{(\tilde{p}+h)^{2}}, b_{5}=$ $-\frac{\tilde{p}}{\tilde{p}+h}, b_{6}=\frac{\beta h \tilde{z}}{(\tilde{p}+h)^{2}}, b_{7}=\frac{\beta \tilde{p}}{\tilde{p}+h}-\mu$

furthermore $T_{2}$ is the equilibrium of Eqs. (16-18), then

1. The interior equilibrium $T_{2}$ of Eqs. (1618) is stable when $\tau<\tau_{k}$ and unstable when $\tau>\tau_{k}$.

2. Eqs. (16-18) undergo a Hopf bifurcation at the interior equilibrium $T_{2}$ when $\tau=$ $\tau_{k}$.

Proof.

The results of the analysis showed that the equilibrium point $T_{2}$ is stable. By giving the time-delay $\tau>0$ will cause the change in the stability of the equilibrium point $T_{2}$. To analyze the stability of the equilibrium point $T_{2}$ with time-delay, we linearize the model (1618) around the equilibrium point $T_{2}$, then we obtain the linearized model

$$
\begin{aligned}
& \frac{d c}{d t}=b_{1} c(t)+b_{2} p(t), \\
& \frac{d p}{d t}=b_{3} c(t)+b_{4} p(t)+b_{5} z(t), \\
& \frac{d z}{d t}=b_{6} p(t-\tau)+b_{7} z(t), \\
& \quad \text { where } b_{1}=\frac{-A \tilde{p}}{(\tilde{c}+1)^{2}}-1, b_{2}=\frac{A}{\tilde{c}+1}, b_{3}= \\
& \frac{B c_{1} \tilde{p}}{\left(\tilde{c}+c_{1}\right)^{2}}, b_{4}=\frac{B \tilde{c}}{\tilde{c}+c_{1}}-2 \tilde{p}-\frac{\tilde{z} h}{(\tilde{p}+h)^{2}}, b_{5}= \\
& \quad-\frac{\tilde{p}}{\tilde{p}+h}, b_{6}=\frac{\beta h \tilde{z}}{(\tilde{p}+h)^{2}}, b_{7}=\frac{\beta \tilde{p}}{\tilde{p}+h}-\mu .
\end{aligned}
$$

Suppose the solution of Eqs. (25-27) is $c(t)=l e^{\lambda \tau}, p(t)=m e^{\lambda \tau}, z(t)=n e^{\lambda \tau}$. 
Substituting Eq. (28) into Eqs. (25-27), then divided $e^{\lambda \tau}$ such that we get $l \lambda=b_{1} l+b_{2} m$,

$m \lambda=b_{3} l+b_{4} m+b_{5} n$,

$n \lambda=b_{6} m e^{-\lambda \tau}+b_{7} n$.

Eqs. (29-31) can be written in the following form.

$$
\left[\begin{array}{c}
l \lambda \\
m \lambda \\
n \lambda
\end{array}\right]=\left[\begin{array}{ccc}
b_{1} & b_{2} & 0 \\
b_{3} & b_{4} & b_{5} \\
0 & b_{6 e^{-\lambda \tau}} & b_{7}
\end{array}\right]\left[\begin{array}{c}
l \\
m \\
n
\end{array}\right] .
$$

So we get the following characteristic equation.

$$
\begin{aligned}
& \left|\begin{array}{ccc}
b_{1}-\lambda & b_{2} & 0 \\
b_{3} & b_{4}-\lambda & b_{5} \\
0 & b_{6} e^{-\lambda \tau} & b_{7}-\lambda
\end{array}\right|=0 \\
& \Leftrightarrow \lambda^{3}+\left(-b_{1}-b_{4}-b_{7}\right) \lambda^{2}+ \\
& \left(b_{1} b_{4}+b_{1} b_{7}+b_{4} b_{7}-b_{2} b_{3}-\right. \\
& \left.b_{5} b_{6} e^{-\lambda \tau}\right) \lambda+\left(b_{2} b_{3} b_{7}-b_{1} b_{4} b_{7}+\right. \\
& \left.b_{5} b_{6} b_{1} e^{-\lambda \tau}\right)=0 .
\end{aligned}
$$

The eigenvalues of the characteristic equation (32) are either real and negative or complex conjugate with negative real parts if only if $-b_{1}-b_{4}-b_{7}>$ $0, b_{1} b_{4}+b_{1} b_{7}+b_{4} b_{7}-b_{2} b_{3}-b_{5} b_{6}>$ 0 and $b_{2} b_{3} b_{7}-b_{1} b_{4} b_{7}+b_{5} b_{6} b_{1}>0$. So with the existence of time-delay, the equilibrium point $T_{2}$ is stable if and only if both conditions and are satisfied. Such that the eigenvalues of the equation (32) we let $\lambda=\mu \pm i \omega$ with $\mu=0$ and $\omega>$ $0(\lambda= \pm i \omega)$. To see the change in the stability of the equation model with time delay, then that eigenvalues are substituted into the equation (32) such that we obtain the roots of the characteristic equation

$\Delta(i \omega, \tau)=b_{1} \omega^{2}+b_{4} \omega^{2}+b_{7} \omega^{2}-$ $b_{5} b_{6} \omega \sin \omega t+b_{2} b_{3} b_{7}-b_{1} b_{4} b_{7} t+$ $b_{5} b_{6} b_{1} \cos \omega t+i\left(-\omega^{3}+b_{1} b_{4} \omega+\right.$ $b_{1} b_{7} \omega+b_{4} b_{7} \omega-b_{2} b_{3} \omega-$ $\left.b_{5} b_{6} \omega \cos \omega t-b_{5} b_{6} b_{1} \sin \omega t\right)$.

Equation (33) is zero if the imaginary and real part are zero, so we obtain $b_{1} \omega^{2}+b_{4} \omega^{2}+b_{7} \omega^{2}+b_{2} b_{3} b_{7}-b_{1} b_{4} b_{7}=$ $\omega b_{5} b_{6} \sin \omega t-b_{5} b_{6} b_{1} \cos \omega t$

and $-\omega^{3}+b_{1} b_{4} \omega+b_{1} b_{7} \omega+b_{4} b_{7} \omega-$ $b_{2} b_{3} \omega=\omega b_{5} b_{6} \cos \omega t+b_{5} b_{6} b_{1} \sin \omega t$.

(35)

Furthermore, eliminating Eqs. (34-35) to $\tau$ by Squaring both sides gives

$$
\begin{aligned}
& b_{1}{ }^{2} \omega^{4}+b_{2}{ }^{2} b_{3}{ }^{2} b_{7}{ }^{2}+2 b_{1} b_{7} \omega^{4}- \\
& 2 b_{1}{ }^{2} b_{4} b_{7} \omega^{2}+2 b_{4} b_{7} \omega^{4}- \\
& 2 b_{4}{ }^{2} b_{1} b_{7} \omega^{2}+2 b_{1} b_{4} \omega^{4}+
\end{aligned}
$$

$$
\begin{aligned}
& 2 b_{7}^{2} b_{2} b_{3} \omega^{2}-2 b_{7}^{2} b_{1} b_{4} \omega^{2}+ \\
& b_{1}{ }^{2} b_{4}{ }^{2} b_{7}{ }^{2}+2 b_{1} b_{2} b_{3} b_{7} \omega^{2}+b_{4}{ }^{2} \omega^{4}- \\
& 2 b_{2} b_{3} b_{7}^{2} b_{1} b_{4}+b_{7}^{2} \omega^{4}+ \\
& 2 b_{4} b_{2} b_{3} b_{7} \omega^{2}=b_{5}{ }^{2} b_{6}{ }^{2} \omega^{2} \sin ^{2} \omega t- \\
& 2 b_{5}{ }^{2} b_{6}{ }^{2} b_{1} \omega \sin \omega t \cos \omega t+ \\
& b_{5}{ }^{2} b_{6}{ }^{2} b_{1}{ }^{2} \cos ^{2} \omega t \\
& \text { and } \\
& -2 b_{1} b_{7} \omega^{4}+2 b_{1}{ }^{2} b_{4} b_{7} \omega^{2}+ \\
& 2 b_{4}{ }^{2} b_{1} b_{7} \omega^{2}+\omega^{6}-2 b_{1} b_{4} b_{2} b_{3} \omega^{2}+ \\
& 2 b_{7}^{2} b_{1} b_{4} \omega^{2}-2 b_{1} b_{2} b_{3} b_{7} \omega^{2}- \\
& 2 b_{4} b_{2} b_{3} b_{7} \omega^{2}+b_{2}{ }^{2} b_{3}{ }^{2} \omega^{2}- \\
& 2 b_{4} b_{7} \omega^{4}+b_{4}{ }^{2} b_{7}{ }^{2} \omega^{2}+b_{1}{ }^{2} b_{4}{ }^{2} \omega^{2}+ \\
& 2 b_{2} b_{3} \omega^{4}+b_{1}{ }^{2} b_{7}{ }^{2} \omega^{2}-2 b_{1} b_{4} \omega^{4}= \\
& b_{5}{ }^{2} b_{6}{ }^{2} \omega^{2} \cos ^{2} \omega t+ \\
& 2 b_{5}{ }^{2} b_{6}{ }^{2} b_{1} \omega \sin \omega t \cos \omega t+ \\
& b_{5}{ }^{2} b_{6}{ }^{2} b_{1}{ }^{2} \sin ^{2} \omega t \text {. }
\end{aligned}
$$

Then adding both Eqs. (35-36) and regrouping by powers of $\omega$, we obtain the following fourth degree polynomial

$\omega^{6}+B \omega^{4}+C \omega^{2}+D=0$,

with

$$
B=b_{1}{ }^{2}+b_{4}{ }^{2}+b_{7}{ }^{2}+2 b_{2} b_{3},
$$

$C=-b_{5}{ }^{2} b_{6}{ }^{2}-2 b_{1} b_{4} b_{2} b_{3}+b_{1}{ }^{2} b_{4}{ }^{2}+$ $2 b_{7}{ }^{2} b_{2} b_{3}+b_{4}{ }^{2} b_{7}{ }^{2}+b_{2}{ }^{2} b_{3}{ }^{2}+b_{1}{ }^{2} b_{7}{ }^{2}$, $D=-2 b_{2} b_{3} b_{7}{ }^{2} b_{1} b_{4}+b_{1}{ }^{2} b_{4}{ }^{2} b_{7}{ }^{2}+$ $b_{2}{ }^{2} b_{3}{ }^{2} b_{7}{ }^{2}-b_{5}{ }^{2} b_{6}{ }^{2} b_{1}{ }^{2}-b_{5}{ }^{2} b_{6}{ }^{2} b_{1}{ }^{2}$. To simplify the calculation suppose $z=$ $\omega^{2}$, so Eq. (37) changes to

$z^{3}+B z^{2}+C z+D=0$,

the root value of equation (38) is determined by Lemma 1 as follows.

Lemma 1. (Ruan)

Define $\xi=B^{2}-3 D$.

(i) If $D<0$, then Eq. (39) has a unique simple positive root.

(ii) If $D \geq 0$ and Eq. (39) $\xi<0$, then Eq. (38) do not have real roots.

(iii) If $D \geq 0$ and $\xi \geq 0$, then Eq. (39) has two positive roots if only if $z=$ $\frac{1}{3}(-B+\sqrt{\xi})>0$ and $h(\bar{z}) \leq 0$.

Suppose that Eq. (39) has a unique simple positive root denoted by $\bar{z}$. Then obtained $\omega_{0}=\sqrt{\bar{z}}$.

Furthermore substituting $\omega_{0}$ into the Eqs. (35-36) and solving for $\tau_{k}$, we get

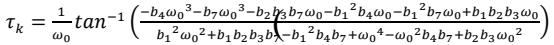

$$
\begin{aligned}
& +\frac{2 k \pi}{\omega_{0}}, k=0,1,2, \cdots
\end{aligned}
$$

Lemma 2

If one of the following is true

(1) $c<0$ and $h^{\prime}\left(\omega_{\text {bif }}\right) \neq 0$; 
(2) $c \geq 0, \Delta>0, \bar{z}>0$ and $h^{\prime}(\bar{z})<0$;

then $\frac{d R e \lambda\left(\tau_{b i f}\right)}{d \tau_{b i f}} \neq 0$,

where $\tau_{k}$ and $\omega_{0}$ defined in Eq. (39).

Further differentiating the Eq. (39) to $\tau$, then we obtain

$$
\begin{gathered}
\Delta(\lambda, \tau)=\lambda^{3}+\left(-b_{1}-b_{4}-b_{7}\right) \lambda^{2}+ \\
\left(b_{1} b_{4}+b_{1} b_{7}+b_{4} b_{7}-b_{2} b_{3}\right) \lambda+ \\
b_{2} b_{3} b_{7}-b_{1} b_{4} b_{7}-b_{5} b_{6} \lambda e^{-\lambda \tau}+ \\
b_{5} b_{6} b_{1} e^{-\lambda \tau}
\end{gathered}
$$

Then

$\frac{d \lambda}{d \tau}=-\frac{\frac{\partial \Delta}{\partial \tau}}{\frac{\partial \Delta}{\partial \lambda}}$

$\left(-b_{5} b_{6} \lambda^{2}+b_{5} b_{6} b_{1} \lambda\right) e^{-\lambda \tau}$

$=\frac{\left(-b_{1}\right.}{3 \lambda^{2}+2\left(-b_{1}-b_{4}-b_{7}\right) \lambda+\left(b_{1} b_{4}+b_{1} b_{7}+b_{4} b_{7}-b_{2} b_{3}\right)+\left(-b_{5} b_{6}+b_{5} b_{c} \tau \lambda-b_{5} b_{6} b_{1} \tau\right)^{-\lambda}}$

Evaluating the real part of this equation at $\tau=\tau_{\text {bif }}$ and setting $\lambda=i \omega_{\text {bif }}$ yield

$\left.\frac{d \lambda}{d \tau}\right|_{\tau=\tau_{\text {bif }}}=\left.\operatorname{Re}\left(\frac{d \lambda}{d \tau}\right)\right|_{\tau=\tau_{\text {bif }}}$

$=\frac{\omega_{b i f}{ }^{2}\left(3 \omega_{b i f}{ }^{4}+2 \omega_{b i f}{ }^{2}\left(b_{1}{ }^{2}+b_{4}{ }^{2}+b_{7}{ }^{2}+2 b_{2} b_{3}\right)\right)}{P_{1}{ }^{2}+Q_{1}{ }^{2}}$

$+\frac{\omega_{b i f}{ }^{2}\left(b_{1}{ }^{2} b_{4}{ }^{2}-2 b_{1} b_{4} b_{2} b_{3}+b_{1}{ }^{2} b_{7}{ }^{2}+b_{4}{ }^{2} b_{7}{ }^{2}+b_{2}{ }^{2} b_{3}{ }^{2}+2 b_{2} b_{3} b_{7}{ }^{2}-b_{5}{ }^{2} b_{6}{ }^{2}\right)}{P_{1}{ }^{2}+Q_{1}{ }^{2}}$

where

$$
\begin{gathered}
P_{1}=-3 \omega_{\text {bif }}^{2}+b_{1} b_{4}+b_{1} b_{7}+b_{4} b_{7}- \\
b_{2} b_{3}+\tau_{b i f}\left(\left(b_{1}+b_{4}+b_{7}\right) \omega_{b i f}{ }^{2}+\right. \\
\left.b_{2} b_{3} b_{7}-b_{1} b_{4} b_{7}\right)-b_{5} b_{6} \cos \omega_{b i f} \tau_{b i f}
\end{gathered}
$$
and

$$
\begin{gathered}
Q_{1}=2\left(-b_{1}-b_{4}-\right. \\
\left.b_{7}\right) \omega_{b i f}+\tau_{b i f}\left(-\omega_{b i f}{ }^{3}+\left(b_{1} b_{4}+\right.\right. \\
\left.\left.b_{1} b_{7}+b_{4} b_{7}-b_{2} b_{3}\right) \omega_{\text {bif }}\right)+ \\
b_{5} b_{6} \sin \omega_{\text {bif }} \tau_{\text {bif }} .
\end{gathered}
$$

Let $\bar{z}=\omega_{\text {bif }}{ }^{2}$, then

$$
\begin{gathered}
h(\bar{z})=x^{3}+\left(b_{1}{ }^{2}+b_{4}{ }^{2}+b_{7}{ }^{2}+\right. \\
\left.2 b_{2} b_{3}\right) x^{2}+\left(b_{1}{ }^{2} b_{4}{ }^{2}-2 b_{1} b_{4} b_{2} b_{3}+\right. \\
\left.b_{1}{ }^{2} b_{7}{ }^{2}+b_{4}{ }^{2} b_{7}{ }^{2}\right) x+\left(b_{2}{ }^{2} b_{3}{ }^{2}+\right. \\
\left.2 b_{2} b_{3} b_{7}{ }^{2}-b_{5}{ }^{2} b_{6}{ }^{2}\right) x+b_{2}{ }^{2} b_{3}{ }^{2} b_{7}{ }^{2}- \\
2 b_{2} b_{3} b_{7}{ }^{2} b_{1} b_{4}+b_{1}{ }^{2} b_{4}{ }^{2} b_{7}{ }^{2}- \\
b_{5}{ }^{2} b_{6}{ }^{2} b_{1}{ }^{2}
\end{gathered}
$$

then

$$
\begin{gathered}
h^{\prime}(\bar{z})=3 x^{2}+2\left(b_{1}{ }^{2}+b_{4}{ }^{2}+b_{7}{ }^{2}+\right. \\
\left.2 b_{2} b_{3}\right) x+b_{1}{ }^{2} b_{4}{ }^{2}-2 b_{1} b_{4} b_{2} b_{3}+ \\
b_{1}{ }^{2} b_{7}{ }^{2}+b_{4}{ }^{2} b_{7}{ }^{2}+b_{2}{ }^{2} b_{3}{ }^{2}+ \\
2 b_{2} b_{3} b_{7}{ }^{2}-b_{5}{ }^{2} b_{6}{ }^{2} .
\end{gathered}
$$

Therefore if $h(\bar{z})<0$ then $h^{\prime}\left(\omega^{2}{ }_{b i f}\right) \neq$ 0.

\section{Simulations at Equilibrium Point $T_{2}$ with Time Delay}

The numerical simulations of the phytoplankton-zooplankton-dissolved oxygen model using Holling II with time delay performed to show the effect of time delay on the equilibrium point $T_{2}$ stability. The parameter values used for the simulations at equilibrium point $T_{2}$ with time delay presented as following.

$B=1,8 ; \beta=1 ; \mu=0,5 ; \gamma=1$;

$h=0,1 ; A=0,53, ; c_{1}=0,8$. Hence obtained

$$
\begin{aligned}
& \tilde{C}=\frac{-1+\sqrt{1+\frac{4 A \mu h}{\beta-\mu}}}{2}=\frac{-1+\sqrt{1+\frac{4.0,53 \cdot 0,5.0,1}{1-0,5}}}{2} \\
& =0,0504358 . \\
& \tilde{p}=\frac{\mu h}{\beta-\mu}=\frac{0,5 \cdot 0,1}{1-0,5}=0,1 . \\
& \tilde{z}=\frac{\beta h\left(\mu B-\mu B \sqrt{1+\frac{4 A \mu h}{\beta-\mu}-\beta B+\beta B} \sqrt{1+\frac{4 A \mu h}{\beta-\mu}}+\mu h-\mu h \sqrt{1+\frac{4 A \mu h}{\beta-\mu}-2 \mu h c_{1}}\right)}{(\beta-\mu)^{2}\left(-1+\sqrt{\left.1+\frac{4 A \mu h}{\beta-\mu}+2 c_{1}\right)}\right.} \\
& =\frac{1.0,1(-0,5.1,8.0,0504358+1.1,1.0,0,0504358-0,5.0,1.0,0504358-0,5.0,1.0,8)}{(1-0,5)^{2}(0,0504358+0,8)} \\
& =0,001357488158 .
\end{aligned}
$$

So obtained the equilibrium point $T_{2}(0,0504358 ; 0,1 ; 0,001357488158)$.

Then from the parameter values, we obtain

$$
\begin{aligned}
& b_{1}=\frac{-A \tilde{p}}{(\tilde{c}+1)^{2}}-1=\frac{-0,53.0,1}{(0,050454358+1)^{2}}-1=-1,048030985 \text {. } \\
& b_{2}=\frac{A}{\tilde{c}+1}=\frac{0,53}{0,0504358+1}=0,5045435777 \text {. } \\
& b_{3}=\frac{B c_{1} \tilde{p}}{\left(\tilde{c}+c_{1}\right)^{2}}=\frac{1,8.0,8.0,1}{(0,0504358+0,8)^{2}}=0,1990950535 \text {. } \\
& b_{4}=\frac{B \tilde{c}}{\tilde{c}+c_{1}}-2 \tilde{p}-\frac{\tilde{z} h}{(\tilde{p}+h)^{2}} \\
& =\frac{1,8,0,0504358}{0,0504358+0,8}-2.0,1-\frac{0,001357488158.0,1}{(0,1+0,1)^{2}}=-0,0966062796 \text {. } \\
& b_{5}=-\frac{\tilde{p}}{\tilde{p}+h}=-\frac{0,1}{0,1+0,1}=-0,5 \text {. } \\
& b_{6}=\frac{\beta h \tilde{z}}{(\tilde{p}+h)^{2}}=\frac{1 \cdot 0,1 \cdot 0,001357488158}{(0,1+0,1)^{2}} \\
& =0,003393720395 \text {. } \\
& b_{7}=\frac{\beta \tilde{p}}{\tilde{p}+h}-\mu=\frac{1 \cdot 0,1}{0,1+0,1}-0,5=0 \text {. } \\
& \omega^{6}+\left(b_{1}{ }^{2}+b_{4}{ }^{2}+b_{7}{ }^{2}+2 b_{2} b_{3}\right) \omega^{4}+ \\
& \left(-b_{5}{ }^{2} b_{6}{ }^{2}-2 b_{1} b_{4} b_{2} b_{3}+b_{1}{ }^{2} b_{4}{ }^{2}+\right. \\
& 2 b_{7}{ }^{2} b_{2} b_{3}+b_{4}{ }^{2} b_{7}{ }^{2}+b_{2}{ }^{2} b_{3}{ }^{2}+ \\
& \left.b_{1}^{2} b_{7}^{2}\right) \omega^{2}+\left(-2 b_{2} b_{3} b_{7}^{2} b_{1} b_{4}+\right. \\
& \left.b_{1}{ }^{2} b_{4}{ }^{2} b_{7}{ }^{2}+b_{2}{ }^{2}{b_{3}}^{2} b_{7}{ }^{2}-b_{5}{ }^{2} b_{6}{ }^{2} b_{1}{ }^{2}\right)= \\
& 0 \\
& \Leftrightarrow \omega^{6}+\left((-1,048030985)^{2}+(-0,0966062796)^{2}+0+\right.
\end{aligned}
$$

For example $z=\omega^{2}$, then equation (39) becomes

$z^{3}+1,30860598 z^{2}-0,0000022485 z-0,000003162571633=0$ $\Leftrightarrow(z+1,308605851)(z+0,001554653882)(z-0,001554525311)=0$

By the condition $\omega_{0}>0$, then selected $\bar{z}=0,001554525311$. Then obtained $\omega_{0}=\sqrt{0,001554525311}=$ $\pm 0,03942746899$. Because of $\omega_{0}>0$, 
then selected $\omega_{0}=0,03942746899$. Then searched the value of $\tau_{k}$ by substituting the values $b_{1}, b_{2}, b_{3}, b_{4}, b_{5}, b_{6}, b_{7}$ and $\omega_{0}$ into the following equation.

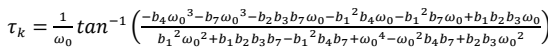

$+\frac{2 k \pi}{\omega_{0}}, k=0,1,2, \cdots$

$\Leftrightarrow \tau_{k}=\frac{1}{0,03942746899}$

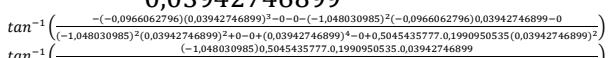

$+\frac{2.0 . \pi}{0,03942746899}$

$\Leftrightarrow \tau_{k}=0,5264846563$.

In this article only discussed the value of time delay when, before and after the delay timeout value in the distance $k=$ 0 .

In addition to the parameters mentioned in Table 2, it is necessary to select the time delay parameters indicated to show changes in equilibrium point stability. In this simulation will be provided three cases to indicate the existence of Hopf bifurcation.

Table 2. Selection of time delay and model stability

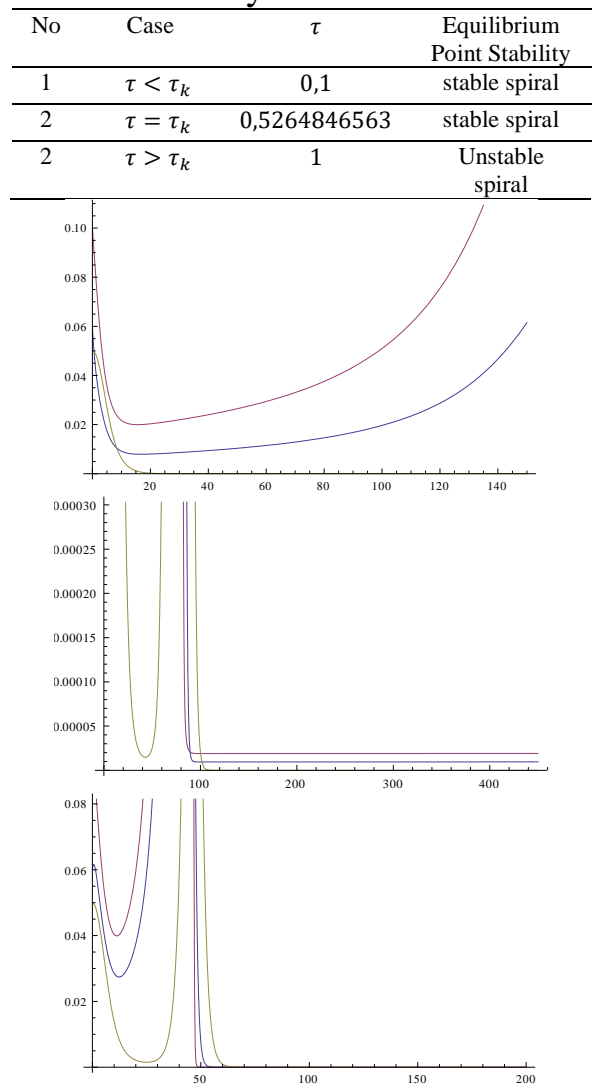

Figure 3. The solution field of predatorprey system at the equilibrium point $T_{2}$ in the case

$$
\tau<\tau_{0}, \tau=\tau_{0} \text { and } \tau>\tau_{0}
$$

\section{Conclusions}

From the above discussion, can be concluded that be based on the nondimensional model, we obtain the following predator-prey model using Holling type II functional response with the time delay in facultative waste stabilization pond.

To analyse the existence of Hopf bifurcation, the predator-prey population dynamics was simulated based on three casses, by increasing the time-delay in the growth rate of the predator population $\left(\tau_{k}\right)$. By chossing an exact parameter value $\left(\tau_{k}\right)$, we can showed the existence of Hopf bifurcation. In the case $\tau=\tau_{k}$ the stable spiral changed into an unstable spiral and also observed the presence of limit cycles. This is known as Hopf bifurcation. Then, to illustrate the model, simulation model was carried out using the Maple 12 software and mathematica 9. The model simulations give the same result with the analysis.

\section{References}

Misra, A. (2010). Modeling the depletion of dissolved oxygen in a lake due to submerged macrophytes Nonlinear Analalysis: Modelling and Control, 15(2), 185- 198.

Misra, A. K. et al. (2011). Modeling the depletion of dissolved oxygen in a lake due to algal bloom: Effect of time delay Advances in Water Resources, 34(10), 1232-1238.

Hull. V. (2008). Modelling dissolved oxygen dynamics in coastal lagoons Ecological Modelling, 211(3), 468480.

Clark, D. N. (2000). Dictionary of Analysis, Calculus and Differential Equations (New York: CRC Press, Boca Raton).

Anton, H. (2005). Elementary Linear Algebra 9th Edition (Singapore: John Wiley \&Sons).

Strogatz, S. H. (2014). Nonlinear Dynamics and Chaos: with Applications to Physics, Biology, Chemistry, and Engineering Second 
Edition: (Massachusetts: Cambridge Perseus Books).

Liao, X. Et al. (2007). Stability of Dynamical Systems Elsevier (Netherlands).

Irwan. (2009). Dynamic Behavior of Competition System Between Tumors with Immune in Delay Differential Equations (Department of Mathematics ITS).
Olsder, G. J. \& Woude, J. W. (2004) Mathematical System Theory (Netherlands: VVSD).

Ruan, S. \& Wei, J. On the zeros of a third degree exponential polynomial with applications to a delayed model for the control of testosterone secretion IMA J. Math. Appl. Med. Biol 18, 41-52. 